\title{
Omega-3 und stille Entzündungen
}

Autor: Volker Schmiedel

\section{ZUSAMMENFASSUNG}

Eine anerkannte Definition der stillen Entzündung existiert bislang nicht. Sie wird u. a. mit einem 2- bis 3-fachen Anstieg von Entzündungsmarkern wie CRP oder TNF- $\alpha$ beschrieben. Epidemiologische Studien belegen aber inzwischen, dass sie Zivilisationskrankheiten wie Diabetes mellitus, Herz-Kreislauf-Erkrankungen, Demenz, nicht-alkoholische Fettleber oder auch chronische Schmerzen und vorschnelle Alterung begünstigen. Hauptursachen sind ein Überkonsum an Omega-6-Fettsäuren und Übergewicht. Eine Diät, die reich an Omega-3-Fettsäuren ist, oder die zusätzliche Gabe von Omega-3-Fettsäuren hat in Studien positive Effekte auf stille Entzündungen ergeben.

Besteht der Verdacht auf eine stille Entzündung kann die subtile Fettsäuren-Analyse ein Ungleichgewicht im Fettsäurenstatus aufdecken. Die Therapie mit Omega-3-Fettsäuren, diätetisch oder als Supplement, hat sich in der Praxis des Autors bewährt.

\section{ABSTRACT}

So far, an accepted definition of the silent inflammation does not exist. It is described among others with a twofold or threefold increase of inflammatory markers like CRP or TNF- $\alpha$. But in the meantime, epidemiologic studies haven proven, that they favor civilization diseases like diabetes mellitus, cardiovascular diseases, dementia, non-alcoholic fatty liver or even chronic pain and premature aging. The main causes are an overconsumption of omega- 6 fatty acids and overweight. In studies, a diet rich in omega- 3 fatty acids or the additional administration of omega-3 fatty acids has shown positive effects on silent inflammations.

If a silent inflammation is suspected, the subtle fatty acid analysis can reveal an imbalance in the fatty acid status. The therapy with omega-3 fatty acids, dietetic or as a supplement, has proven its worth in the medical practice of the author.

Keywords

Silent inflammation, low-grade inflammation, omega-3 fatty acids

Stille Entzündung, silent inflammation, low-grade inflammation, Omega-3-Fettsäuren

Bei Wikpedia (deutsch- und englischsprachig) sucht man nach den Stichworten „stille Entzündung“, „silent inflammation“ oder „low-grade inflammation“ immer noch vergeblich. Dies ist erstaunlich, da sich bei Pubmed immerhin 452 Artikel finden lassen, die „low-grade inflammation “ sogar im Titel tragen. Dabei häufen sich Hinweise, dass zahlreichen Krankheiten durch stille Entzündungen Vorschub geleistet wird. Hier sollen einige Grundlagen zu stillen Entzündungen sowie praktische Diagnostik- und Therapiehinweise vorgestellt werden.

\section{Was sind eigentlich „stille Entzündungen"?}

Zunächst einmal: Was sind eigentlich Entzündungen? „Richtige Entzündungen“ zeichnen sich durch die klassischen Entzündungszeichen rubor, calor, dolor, tumor und/ oder functio laesa sowie eine erhöhte BSG über $10 \mathrm{~mm} / \mathrm{h}$ und/oder ein CRP über $5 \mathrm{mg} / \mathrm{dl}$ aus. Eine allseits anerkannte Definition der stillen Entzündung existiert bislang nicht. Eine von mir gefundene Definition [16] beschreibt einen 2- bis 3-fachen Anstieg von Entzündungsmarkern wie CRP oder TNF- $\alpha$. Doch was sind eigentlich die „Normalwerte“ 


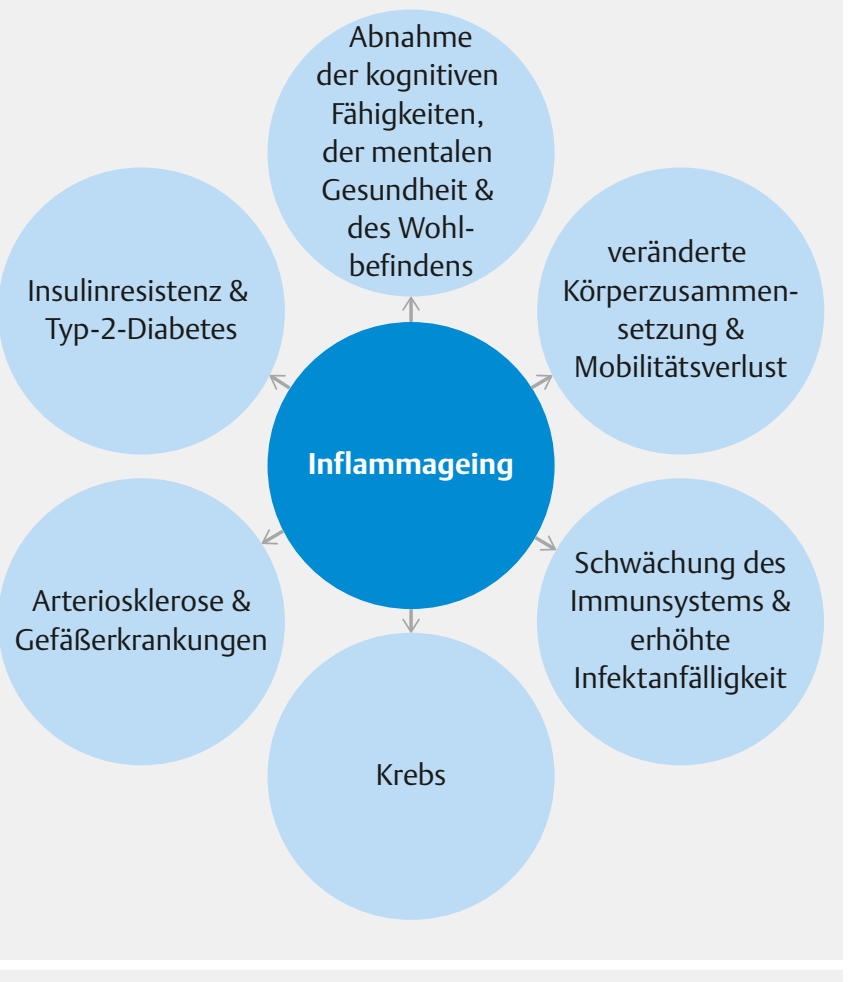

- Abb. 2 Stille Entzündungen aus Auswirkungen auf altersabhängige Minderfunktionen des Organismus.

dieser Entzündungsmarker? Der Grenzwert $5 \mathrm{mg} / \mathrm{dl}$ bei der CRP beschreibt lediglich die Grenze zur echten Entzündung. Wirklich Gesunde weisen meist eine CRP von unter 1 , ja sogar unter $0,5 \mathrm{mg} / \mathrm{dl}$ auf. Ich selbst definiere für mich eine stille Entzündung als ein CRP von 1-5 mg/dl bei Abwesenheit von akuten Entzündungszeichen oder einer akuten Entzündung (z. B. grippaler Infekt) in den letzten Wochen.

\section{Folgen stiller Entzündungen}

Ein Großteil der heutigen Zivilisationskrankheiten wird durch stille Entzündungen begünstigt. Dazu zählen Diabetes mellitus, Krebs, Herzinfarkt, Schlaganfall, Demenz, psychische Krankheiten, chronisch-obstruktive Lungenerkrankung (COPD), Allergien, Arteriosklerose, nicht-alkoholische Fettleber, chronische Schmerzen und vorschnelle Alterung und Degeneration.

Einige Forscher sprechen seit kurzem vom sog. „Inflammageing“, was ausdrücken soll, dass Entzündungs- und Alterungsprozesse miteinander Hand in Hand gehen. Hier spielen insbesondere stille Entzündungen eine große Rolle. Diese sind damit Wegbereiter für kognitiven Verfall, Insulinresistenz, Mobilitätsverlust, Arteriosklerose, Einschränkungen des Immunsystems und Krebs ( $\mathbf{A}$ bb. 1). Es werden Studien vorgelegt, die die Beeinflussung stiller Entzündungen durch Omega-3-Fettsäuren, Präbiotika, Probiotika, Antioxidantien und Polyphenole aufzeigen.
Außerdem wird die Rolle der Mikrobiota bei Entzündungen diskutiert [3].

\section{Ursachen stiller Entzündungen}

Hauptursachen sind ein Überkonsum an Omega-6-Fettsäuren und Übergewicht. Ein hoher AA/EPA-Quotient fördert Entzündungen. Dieser setzt sich aus zu hohem Omega-6- und zu geringem Omega-3-Fettsäure-Konsum zusammen. Da das Fettgewebe nicht nur ein Energiespeicher, sondern zugleich auch eine höchst aktive Drüse ist (produziert bestimmte Hormone wie z. B. Östrogen und setzt Entzündungsbotenstoffe wie Zytokine und Interleukine frei), fördert Übergewicht ebenfalls stille Entzündungen.

Eine Studie mit 262 Patienten, die in 4 BMI-Kategorien $(<25,25-30,30-40,>40)$ eingeteilt wurden, fand heraus, dass es eine positive Korrelation zwischen BMI und den Entzündungsmarkern CRP und IL-6 gibt [8].

Bei der Fettverteilung ist besonders das viszerale Fettgewebe zu beachten. So konnte ein klarer Zusammenhang zwischen Waist-to-hip-ratio und Entzündungsmarkern sowieso Insulinresistenz gefunden werden [4].

\section{Blutuntersuchung: Entzündungsmarker und AA/EPA-Quotient}

Ob Patienten an einer stillen Entzündung leiden, lässt sich durch die Beurteilung der Entzündungsmarker relativ leicht herausfinden. Viele Ärzte schenken den Entzündungsmarkern aber leider keine Beachtung. Therapeuten, die ganzheitlich arbeiten, können seit kurzem auch eine Fettsäure-Analyse verwenden. Hierbei werden Omega-3-, Omega-6-, Omega-9-Fettsäure-Werte und Werte der gesättigten und Transfettsäuren gemessen.

Außerdem kann der AA/EPA-Quotient bestimmt werden. AA ist die wichtigste entzündungsfördernde Omega-6Fettsäure Arachidonsäure und EPA die wichtigste entzündungshemmende Omega-3-Fettsäure Eicosapentaensäure.

Es wird also das Verhältnis dieser beiden Fettsäuren zueinander bestimmt. Je geringer dieses ist, desto geringer die Neigung zu Entzündungen. Das AA/EPA-Verhältnis ist daher ein guter Marker für die Entzündungsneigung, zeigt Entzündungen allerdings nicht direkt an.

\section{Der normale bzw. ideale AA/EPA-Quotient}

Optimalerweise sollte der Quotient höchstens 2,5 betragen. Vermutlich hatten die meisten Menschen vor etwa 200 Jahren einen solchen Wert. Steinzeitmenschen vor Tausenden von Jahren oder die Inuit heutzutage, wenn 
sie sich traditionell ernähren, haben einen Quotient von unter 1.

Der Zusammenhang zwischen der Zusammensetzung der Fettsäuren und Entzündungsmarkern darf inzwischen als gesichert angesehen werden. Von 744 kanadischen Erwachsenen (Cree-Indianer an der James Bay) hatten $47 \%$ ein erhöhtes hsCRP (>3 mg/l). DPA (eine Intermediärstufe zwischen EPA und DHA) war dabei invers korreliert zu hsCRP und TNF- $\alpha$. Die Forscher folgern daraus, dass stille Entzündungen häufig auftreten, aber umso geringer ausgeprägt sind, je besser die Versorgung mit Omega-3-Fettsäuren ist [11].

In einem Review wurden die Veränderungen der menschlichen Ernährung besonders bezüglich der Fette beschrieben. Die Wissenschaftler präsentieren dabei Beweise für den Zusammenhang einer Überversorgung mit Omega6- und einer Unterversorgung mit Omega-3-Fettsäuren und stillen Entzündungen. Hohe Omega-6/3-Quotienten führen danach zu einer vermehrten Synthese von proinflammatorischen Zytokinen und Adipositas sowie Osteoporose [9].

In einer anderen Arbeit schildern die Autoren epidemiologische Beweise für den Zusammenhang zwischen Omega-3-Fettsäuren und niedrigeren Entzündungs- und besseren metabolischen Parametern. Auch wenn nach Ansicht der Autoren die Datenlage bezüglich Interventionsstudien noch inkonsistent erscheint, favorisieren sie die Zufuhr von Omega-3-Fettsäuren, um bei Patienten mit metabolischen Problemen die stille Entzündung zu mindern [18].

\section{Stille Entzündung und Krankheit}

Gibt es denn Hinweise darauf, dass stille Entzündungen tatsächlich Krankheiten Vorschub leisten? Hier eine Auswahl von epidemiologischen Studien. Bei 20337 Erwachsenen ohne akute Entzündungszeichen erstellte man einen Score für stille Entzündungen. Als Biomarker flossen darin ein: CRP-Wert, Leukozyten sowie der Quotient aus Granulozyten und Lymphozyten. In 7,6 Jahren Untersuchungszeitraum kam es zu insgesamt 837 Todesfällen. Das höchste Quartil des Entzündungsscores wies eine um 44 \% höhere Gesamtsterblichkeit im Vergleich zum niedrigsten Quartil auf. Bei Patienten mit Diabetes mellitus Typ 2 kam es unter denen mit höheren Entzündungsmarkern sogar fast zu einer Verdreifachung $(H R=2,90)$ des Sterberisikos. Bei Patienten mit KHK war es mehr als verdoppelt $(H R=2,48)$. Stille Entzündungen erwiesen sich damit als signifikanter unabhängiger Risikofaktor für die Gesamtsterblichkeit, besonders aber bei Risikopersonen [2].

15754 dänische Blutspender füllten einen Fragebogen aus. Stille Entzündung wurde als gegeben angesehen, wenn ein CRP zwischen 3 und $10 \mathrm{mg} / \mathrm{l}$ vorlag. Innerhalb des Untersuchungszeitraums von 3,4 Jahren kam es zu 571 stationären Aufenthalten wegen Infektion. Das Risiko für einen Krankenhausaufenthalt war bei Vorliegen einer stillen Entzündung um 60 \% erhöht [10].

An der „Malmö Diät und Krebs Kohortenstudie“ 1991 nahmen insgesamt 8513 Frauen zwischen 55 und 73 Jahren teil. Nach 20 Jahren wurden 459 neue Brustkrebsfälle diagnostiziert. Das niedrigste Tertil von TNF- $\alpha$ hatte ein relatives Risiko von 0,65 ( $p=0,04)$, das höchste Tertil von IL-1 eines von $1,71(p=0,01)$. Die Forscher folgerten daraus, dass stille Entzündungen das Risiko für postmenopausalen Brutkrebs erhöhen [7].

\section{Was bewirkt die Intervention mit Omega-3-Fettsäuren?}

Auch hierzu gibt es mittlerweile eine Vielzahl an Studien. 30 gesunde Frauen unter Hormonersatztherapie wurden randomisiert auf drei Gruppen verteilt und erhielten 5 Wochen täglich $14 \mathrm{~g}$ Distelöl, $7 \mathrm{~g}$ Distel- und $7 \mathrm{~g}$ Fischöl oder 14 gischöl. In beiden Fischölgruppen kam es zu einer signifikanten Absenkung des Entzündungsmarkers CRP und des Entzündungsmediators IL-6. In der Fischölgruppe lagen Triglyceride und das Triglycerid/HDL-Verhältnis signifikant niedriger. Die Autoren folgern daraus, dass eine Substitution mit Fischöl bei postmenopausalen Frauen aufgrund der antiinflammatorischen Wirkung das kardiovaskuläre Risiko zu senken vermag [6].

35 Männer im Alter von durchschnittlich 55 Jahren mit Niereninsuffizienz unter Hämodialyse erhielten 8 Wochen lang 2,4 g EPA/DHA. Seruminsulin, HOMA-IR, TNF- $\alpha$, IL-6 und hsCRP waren am Ende des Versuchs hochsignifikant niedriger. Die Forscher schlossen daraus, dass Omega-3-Fettsäuren einen günstigen Effekt auf stille Entzündungen und auf die Insulinresistenz haben [17].

In einer kontrollierten, doppelblinden Crossover-Studie erhielten 48 Männer und 106 Frauen mit abdomineller Adipositas und stiller Entzündung 2,7g EPA, 2,7g DHA oder Maiskeimöl jeweils getrennt durch eine 9-wöchige Auswaschphase. Nur in den EPA- und DHA-Gruppen kam es zu einer Senkung von CRP, IL-6 und TNF- $\alpha$, wobei sich DHA tendenziell sogar als stärker erwies [1].

Eine sehr differenzierte und umfassende Studie widmet sich der Steatosis hepatosis, bei der Entzündungen zum fibrotischen und später zirrhotischen Umbau beitragen. 78 Patienten mit nicht-alkoholischer Steatohepatosis erhielten Omega-3-Fettsäuren oder Placebo. Nach 6 Monaten waren die Leber-, Lipid- und Entzündungsparameter ALT, AST, Triglyceride, Gesamtcholesterin, CRP signifikant reduziert. Außerdem waren der Marker für nitro-oxidativen Stress Malondialdehyd sowie die Fibrosemarker TypIV-Kollagen und Pro-Kollagen-III-Peptid signifikant reduziert ( $\triangleright$ Tab. 1). Weiterhin verbesserten sich histologisch 
Steatosegrad und Fibrosierungsgrad ( $\vee$ Tab. 2 ). Die Forscher schlussfolgerten, dass eine vermehrte Zufuhr von Omega-3-Fettsäuren bei NASH die Progression zu verzögern vermag [12].

In einer Ernährungsstudie erhielten 30 übergewichtige Typ-II-Diabetiker 24 Wochen lang eine proteinreiche Diät mit niedrigem glykämischen Index, die mit Omega-3-Fettsäuren ergänzt wurde. Das HbA1c sank hierunter von 63 auf $59(p=0,033)$ bzw. $56 \mathrm{mmol} / \mathrm{l}(p=0,001)$ nach 12 bzw. 24 Wochen. Damit einhergehend sank das hsCRP ebenfalls signifikant $(p=0,039)$. Der Taillenumfang wurde mit $116 \mathrm{~cm}$ auf $112,7 \mathrm{~cm}$ ebenfalls signifikant geringer $(p=0,049)$, was immerhin etwa einem Loch enger im Gürtel entspricht. Schlussfolgerung der Autoren: Eine solche Diät zusammen mit Omega-3-Fettsäuren mindert Entzündung und verbessert Stoffwechselparameter bei Typ-II-Diabetikern [15].

In einer anderen Ernährungsstudie wurden Patienten mit metabolischem Syndrom randomisiert in zwei Diätinterventions- und eine Kontrollgruppe eingeteilt. 44 Probanden erhielten eine Healthy Diet (fetter Fisch, Heidelbeeren), 42 eine WGED (whole grain enriched diet, also vermehrt Vollkornprodukte) und 45 stellten die Kontrollgruppe dar. In der Healthy Diet sank das hsCRP um 17 und in der WGED um 27\%. Die Autoren folgern daraus, dass die Kombination von fetten Fischen, Heidelbeeren und Vollkornprodukten stille Entzündungen und damit das Risiko von Diabetes bei Risikopersonen vermindern könnten [14].

Depressive Patienten bekommen leichter KHK und KHKPatienten sind depressiver als Gesunde. Wissenschaftler postulieren Omega-3-Fettsäuren als Bindeglied zwischen Depression und koronarer Herzkrankheit, welche überzufällig häufig gleichzeitig vorliegen. Sie vermuten, dass Omega-3-Fettsäuren eine vielversprechende Therapie für kardiovaskuläre Erkrankungen und Depression sein könnten - aufgrund der antiinflammatorischen, kardioprotektiven und neuroprotektiven Effekte [5].

Zum Schluss sei auch noch eine „negative Studie“ zitiert. 60 gesunde Freiwillige wurden randomisiert auf drei Gruppen verteilt. Sie erhielten 6,6, 2,0 g Omega-3-Fettsäuren oder Olivenöl. Nach 12 Wochen wurden keine Unterschiede zwischen den einzelnen Gruppen bezüglich CRP gemessen.

Anmerkung des Autors: Ich zitiere ganz bewusst diese Studie, nicht weil sie beweist, dass Omega-3-Fettsäuren doch nicht antientzündlich wirken, sondern um aufzuzeigen, dass Studien klug konzipiert sein sollten. In dieser Studie hatten die Probanden einen CRP-Ausgangswert von $0,78 \mathrm{mg} / \mathrm{dl}$, sie waren also völlig entzündungsfrei, ja sie hatten noch nicht einmal Hinweise auf eine stille Entzündung. Was soll denn da noch gesenkt werden? Der zweite gravierende Fehler: Als „Placebo“ wurde ein Olivenöl

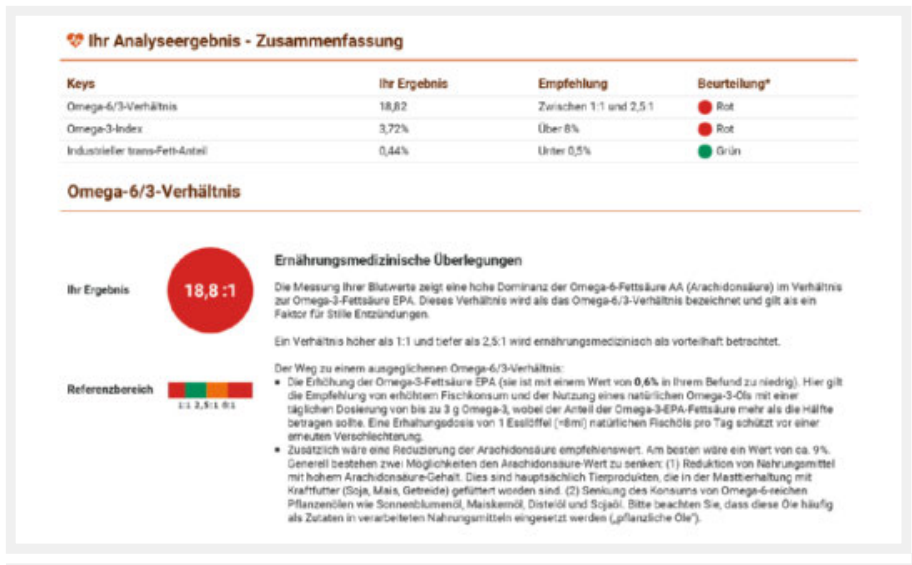

- Abb. 3 Hoher AA/EPA-Quotient von über 18 bei einer Patientin mit Multipler Sklerose.

- Tab. 1 Laborwerte bei Patienten mit NASH ohne und mit Omega-3Fettsäuren nach 6 Monaten.

\begin{tabular}{|l|l|l|l|}
\hline Parameter & Placebogruppe & Omega-3-Gruppe & p-Wert \\
\hline$n$ & 39 & 39 & \\
\hline ALT (U/I) & $80,4 \pm 7,6$ & $67,8 \pm 5,3$ & $<0,01$ \\
\hline AST (U/I) & $75,6 \pm 5,8$ & $60,3 \pm 6,8$ & $<0,01$ \\
\hline Triglyceride (mmol/I) & $2,4 \pm 0,4$ & $1,8 \pm 0,3$ & 0,015 \\
\hline Cholesterin (mmol/I) & $5,2 \pm 0,6$ & $4,7 \pm 0,3$ & 0,040 \\
\hline CRP (mg/l) & $9,2 \pm 0,8$ & $7,6 \pm 0,4$ & 0,045 \\
\hline MDA (nmol/ml) & $0,6 \pm 0,1$ & $0,4 \pm 0,2$ & 0,048 \\
\hline Typ-IV-Kollagen (ng/ml) & $4,6 \pm 0,7$ & $3,5 \pm 0,5$ & 0,023 \\
\hline P-III-P (U/ml) & $0,8 \pm 0,2$ & $0,5 \pm 0,3$ & 0,039 \\
\hline
\end{tabular}

- Tab. 2 Histologie bei Patienten mit NASH ohne und mit Omega-3Fettsäuren nach 6 Monaten.

\begin{tabular}{|l|l|l|l|}
\hline Parameter & Placebogruppe & Omega-3-Gruppe & p-Wert \\
\hline Steatosegrad & $1,08 \pm 0,1$ & $1,4 \pm 0,2$ & 0,032 \\
\hline $\begin{array}{l}\text { Grad der nekrotischen } \\
\text { Entzündung }\end{array}$ & $1,5 \pm 0,1$ & $1,1 \pm 0,1$ & 0,017 \\
\hline Fibrosegrad & $1,6 \pm 0,3$ & $1,1 \pm 0,2$ & 0,020 \\
\hline Ballooning Score & $1,6 \pm 0,2$ & $1,0 \pm 0,2$ & 0,015 \\
\hline
\end{tabular}

gewählt. Ein gutes, kaltgepresstes Olivenöl enthält aber zahlreiche Polyphenole, von denen einige wie etwa das Oleocanthal eine sehr gute antiinflammatorische Wirkung aufweisen [13].

\section{Also möglichst viele Fische essen?}

Fische weisen wegen ihres Gehalts an Omega-3-Fettsäuren nutritive Vorteile auf. Doch ist ein hoher Konsum wegen der Belastung an Schwermetallen und lipidlöslichen 
Fettsäure-Werte (in \% von den gesamten Fettsäuren)

\begin{tabular}{|c|c|c|}
\hline Omega-3-Fettsäuren & Ihre Werte & Referenzwerte* \\
\hline Alpha-Linolensäure (ALA, 18:3 $\omega 3$ ) & 0,80 & 0,36 \\
\hline Eicosapentaensäure (EPA, 20:5 $\omega 3$ ) & 0,56 & 3,78 \\
\hline Docosapentaensäure (DPA, 22:5 $\omega 3$ ) & 1,27 & 2,03 \\
\hline Docosahexaensäure (DHA, 22:6 $\omega 3$ ) & 2,52 & 6,00 \\
\hline Total Omega-3 & 5,15 & 12,17 \\
\hline Omega-6-Fettsäuren & Ihre Werte & Referenzwerte* \\
\hline Linolsäure (LA, 18:2 $\omega 6)$ & 20,35 & 16,72 \\
\hline Gamma-Linolensäure (GLA, 18:3 $\omega 6$ ) & 0,11 & 0,14 \\
\hline Eicosadiensäure (C20:2 $\omega 6)$ & 0,17 & 0,20 \\
\hline Dihomo-y-Linolensä (DGLA, 20:3 $\omega 6$ ) & 1,56 & 1.29 \\
\hline Arachidonsäure (AA, 20:4 $\omega 6)$ & 10,54 & 8,94 \\
\hline Docosatetraensäure (DTA, 22:4 $\omega 6$ ) & 1,06 & 0,76 \\
\hline $\mathrm{C} 22: 5 \omega 6$ & 0,31 & 0,25 \\
\hline Total Omega-6 & 34,10 & 28,30 \\
\hline Omega-7-Fettsäuren & Ihre Werte & Referenzwerte* \\
\hline Palmitolein $(16: 1 \omega 7)$ & 0,73 & 0,70 \\
\hline Omega-9-Fettsäuren & Ihre Werte & Referenzwerte* \\
\hline Ölsăure (18:1 $\omega 9)$ & 20,33 & 18,74 \\
\hline Gadoleinsäure (20:1 $\omega 9)$ & 0,23 & 0,21 \\
\hline Nervonsäure $(24: 1 \omega 9)$ & 0,55 & 0,38 \\
\hline Total Omega-9 & 21,11 & 19,33 \\
\hline Transfettsäuren & Ihre Werte & Referenzwerte* \\
\hline Trans-Palmitolein (16:1 $\omega 7 \mathrm{t})$ & 0,18 & 0,13 \\
\hline Trans-Olsäure (18:1t) & 0,62 & 0,20 \\
\hline Trans-Linolsäure $(18: 2 \omega 6 \mathrm{tt} / \mathrm{tc} / \mathrm{ct})$ & 0,13 & 0,17 \\
\hline Total Transfettsäuren & 0,93 & 0,50 \\
\hline Gesättigte Fettsäuren & Ihre Werte & Referenzwerte* \\
\hline Myristinsäure (14:0) & 1,16 & 0,72 \\
\hline Palmitinsäure (16:0) & 23,08 & 24,0 \\
\hline Stearinsäure (18:0) & 12,82 & 13,15 \\
\hline C20:0 & 0,17 & 0,16 \\
\hline C22:0 & 0,37 & 0,19 \\
\hline Lignocerinsäure (24:0) & 0,38 & 0,37 \\
\hline Total gesăttigte Fettsäuren & 37,98 & 38,59 \\
\hline
\end{tabular}

- Abb. 4 Einzelne Fettsäuren bei der gleichen Patientin mit sehr niedrigem EPA von 0,62\% (>3\% wünschenswert) bei gleichzeitig hoher AA von $11,25 \%$ (<9\% wünschenswert).

Toxinen nicht vielleicht problematisch? Das Health 2000 Survey repräsentiert mit 1173 Teilnehmern die allgemeine finnische Bevölkerung. Die Fishermen Study repräsentiert mit 255 Teilnehmern einen Teil der finnischen Bevölkerung mit sehr hohem Fischkonsum. In beiden Populationen sanken die Triglyceride mit steigendem Omega-3-Fettsäuregehalt im Blut. Insulinresistenz, CRP, TNF- $\alpha$ und IL- 6 sanken in der allgemeinen Bevölkerung mit steigendem Omega-3-Gehalt. In der Population der Fischer stiegen jedoch die Insulinresistenz und die arterielle Steifigkeit mit steigenden PCB- und Quecksilberspiegeln. Die Wissenschaft- ler folgerten daraus, dass die toxikologischen Belastungen durch hohen Fischkonsum die nützlichen Effekte der Omega-3-Fettsäuren konterkarieren [19].

Anmerkung des Autors: In der Konsequenz sollte dann der Fischkonsum nicht sehr hoch sein (möglicherweise 1-2 Portionen Seefisch pro Woche) und die Zufuhr weitgehend durch schadstofffreies Fischöl gedeckt werden, um die nützlichen Effekte der Omega-3-Fettsäuren zu generieren und die nachteiligen Toxinbelastungen durch die Fische zu vermeiden. 


\section{Praktisches Vorgehen bei stillen Ent- zündungen}

Wenn ich bei einer Laboruntersuchung eine stille Entzündung finde und etwa eine familiäre Belastung für entzündungsabhängige Krankheiten wie KHK oder Krebs besteht, der Patient vielleicht schon selbst eine solche Krankheit wie metabolisches Syndrom oder Depression aufweist, messe ich die Omega-3- und -6-Fettsäuren mittels einer subtilen Fettsäureanalyse. Bei erhöhtem Risiko für eine entzündungsabhängige Krankheit gebe ich so viel Omega-3-Fettsäuren, dass ein AA/EPA-Quotient von 3-5 erzielt wird. Liegt aber schon eine solche Krankheit vor, möchte ich den Patienten in einen Bereich unter 2,5 bringen. Da die Fettsäuren am besten in der Erythrozytenmembran gemessen werden (was eine stabilere Messung als die durch die aktuelle Ernährung sehr störanfällige Plasmabestimmung darstellt), sollte eine Kontrollmessung erst nach etwa 3 Monaten erfolgen. Die dann gemessenen Werte sind ziemlich konstant, wenn es zu keiner Änderung der Fettsäurezufuhr über die Nahrung oder Supplemente kommt.

\section{Welche Omega-3-Fettsäuren sind zu empfehlen?}

Aufgrund geringer toxischer Werte sind hochwertige Fischöl-Präparate zur Einstellung des optimalen Quotienten zu empfehlen. Es sollten mindestens $2 \mathrm{~g}$ Omega-3Fettsäuren täglich eingenommen werden, was etwa 15 konventionellen Fischölkapseln (0,5 g Fischöl) oder 1 EL Fischöl entspricht. Alternativ stehen für Veganer/Vegetarier mittlerweile gute Algenölpräparate zur Verfügung.

\section{Fazit}

Selbst wenn stille Entzündungen zunächst nicht schmerzen und nicht wahrgenommen werden, sind sie sehr gefährlich und sollten nicht ignoriert werden. Sie können die Entstehung von Krankheiten, die entzündungsabhängig sind, begünstigen. Die Therapie stiller Entzündungen stellt also eine primäre Prävention im besten Sinne dar. Die Diagnostik von Fettsäureungleichgewichten und die Therapie mit Omega-3-Fettsäuren möchte ich in meinem praktischen Alltag nicht mehr missen.

Interessenkonflikt

Der Autor hat in den letzten Jahren Vorträge zum Thema für die Firmen Biogena, Hepart, Loges und San Omega gehalten.

Autor

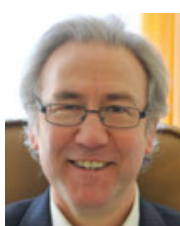

\section{Volker Schmiedel}

war von 1996-2015 Chefarzt der Inneren Abteilung der Habichtswaldklinik Kassel. Seit 2016 ist er als Arzt im ganzheitlichen Ambulatorium Paramed in Baar (Schweiz) tätig. Er war viele Jahre Fortbildungsleiter für „Naturheilverfahren“ der Medizinischen

Woche, ist Mitherausgeber der Zeitschrift „Erfahrungsheilkunde" und des "Leitfaden Naturheilkunde“ sowie Autor zahlreicher weiterer naturheilkundlicher Bücher für Therapeuten und Laien. 
Korrespondenzadresse

Dr. med. Volker Schmiedel, M.A.

Facharzt für Physikalische und Rehabilitative Medizin, Naturheilverfahren, Homöopathie

Paramed AC

Kompetenzzentrum für Ganzheitsmedizin

Haldenstr. 1

CH-6340 Baar

E-Mail:v.schmiedel@paramed.ch

\section{Zum Weiterlesen}

Schmiedel V, Augustin M, Hrsg. Leitfaden Naturheilkunde. 7. Aufl. München: Elsevier; 2017

Schmiedel V. Omega-3 - Öl des Lebens. Lenzburg: FONA: 2018

Schmiedel V. Vitamine, Mineralstoffe und Spurenelemente. Ernährung, Diagnostik und Nährstofftherapie. Stuttgart: Thieme; (voraussichtliches Erscheinen 1/2019)

Literatur

[1] Allaire J, Couture P, Leclerc M et al. A randomized, crossover, head-to-head comparison of eicosapentaenoic acid and docosahexaenoic acid supplementation to reduce inflammation markers in men and women: the Comparing EPA to DHA (ComparED) Study. Am J Clin Nutr 2016; 104 (2): 280-287. doi: 10.3945/ajcn.116.131896

[2] Bonaccio M, Di Castelnuovo A, Pounis G et al. A score of low-grade inflammation and risk of mortality: Prospective findings from the Moli-sani Study. Haematologica 2016; 101 (11): 1434-1441

[3] Calder PC, Bosco N, Bourdet-Sicard R et al. Health relevance of the modification of low grade inflammation in ageing (inflammageing) and the role of nutrition. Ageing Res Rev 2017; 40: 95-119. doi: 10.1016/j.arr.2017.09.001

[4] Caminiti C, Armeno M, Mazza CS. Waist-to-height ratio as a marker of low-grade inflammation in obese children and adolescents. J Pediatr Endocrinol Metab 2016; 29 (5): 543-551. doi: 10.1515/jpem2014-0526

[5] Chang JP, Chen YT, Su KP. Omega-3 polyunsaturated fatty acids ( $n-3$ PUFAs) in cardiovascular diseases (CVDs) and depression: the missing link? Cardiovasc Psychiatry Neurol 2009; doi: $10.1155 / 2009 / 725310$

[6] Ciubotaru I, Lee YS, Wander RC. Dietary fish oil decreases C-reactive protein, interleukin-6, and triacylglycerol to HDL-cholesterol ratio in postmenopausal women on HRT. J Nutr Biochem 2003; 14 (9): 513-521. doi: 10.1016/S09552863(03)00101-3

[7] Dias JA, Fredrikson GN, Ericson $U$ et al. Low-grade inflammation, oxidative stress and risk of invasive post-menopausal breast cancer - a nested case-control study from the Malmö Diet and Cancer Cohort. PLoS One 2016; 11 (7): e0158959. doi: 10.1371/journal.pone.0158959

[8] Ebron K, Andersen C], Aguilar D et al. A larger body mass index is associated with increased atherogenic dyslipidemia, insulin resistance, and lowgrade inflammation in Individuals with Metabolic Syndrome. Metab Syndr Relat Disord 2015; 13 (10): 458-464. doi: 10.1089/met.2015.0053
[9] Ilich JZ, Kelly OJ, Kim Y et al. Low-grade chronic inflammation perpetuated by modern diet as a promoter of obesity and osteoporosis. Arh Hig Rada Toksikol 2014; 65 (2): 139-148. doi: 10.2478/10004-1254-65-2014-2541

[10] Kaspersen KA, Dinh KM, Erikstrup LT et al. Low-grade inflammation is associated with susceptibility to infection in healthy men: Results from the Danish Blood Donor Study (DBDS). PLoS One 2016; 11 (10): e0164220. doi: 10.1371/ journal.pone. 0164220

[11] Labonté MÈ, Dewailly E, Lucas M et al. Association of red blood cell n-3 polyunsaturated fatty acids with plasma inflammatory biomarkers among the Quebec Cree population. Eur J Clin Nutr 2014; 68 (9): 1042-1047. doi: 10.1038/ ejcn.2014.125

[12] Li YH, Yang LH, Sha KH et al. Efficacy of poly-unsaturated fatty acid therapy on patients with nonalcoholic steatohepatitis. World J Gastroenterol 2015; 21 (22): 7008-7013. doi: 10.3748/wjg.v21.i22.7008

[13] Madsen T, Christensen JH, Blom M et al. The effect of dietary $\mathrm{n}-3$ fatty acids on serum concentrations of $\mathrm{C}$-reactive protein: a dose-response study. Br J Nutr 2003; 89 (4): 517-522

[14] de Mello VD, Schwab U, Kolehmainen M et al. A diet high in fatty fish, bilberries and wholegrain products improves markers of endothelial function and inflammation in individuals with impaired glucose metabolism in a randomised controlled trial: the Sysdimet study. Diabetologia 2011; 54 (11): 2755-2767. doi: 10.1007/s00125-011-2285-3

[15] Moosheer SM, Waldschütz W, Itariu BK et al: A proteinenriched low glycemic index diet with omega-3 polyunsaturated fatty acid supplementation exerts beneficial effects on metabolic control in type 2 diabetes. Prim Care Diabetes 2014; 8 (4): 308-314. doi: 10.1016/j.pcd.2014.02.004

[16] Petersen AM, Pedersen BK. The anti-inflammatory effect of exercise. J Appl Physiol 2005; 98 (4): 1154-1162. doi: 10.1152/japplphysiol.00164.2004

[17] Rasic-Milutinovic Z, Perunicic G, Pljesa S et al. Effects of N-3 PUFAs supplementation on insulin resistance and inflammatory biomarkers in hemodialysis patients. Renal failure 2007; 29: 321-329. doi: 10.1080/08860220601184092

[18] Robinson LE, Mazurak VC. N-3 polyunsaturated fatty acids: relationship to inflammation in healthy adults and adults exhibiting features of metabolic syndrome. Lipids 2013; 48 (4): 319-332. doi: 10.1007/s11745-013-3774-6

[19] Turunen AW, Jula A, Suominen AL et al. Fish consumption, omega- 3 fatty acids, and environmental contaminants in relation to low-grade inflammation and early atherosclerosis. Environ Res 2013; 120: 43-54. doi: 10.1016/j. envres.2012.09.007 Bibliografie

Bibliografie

DOI https://doi.org/10.1055/a-0647-0080

EHK 2018; 67: 198-204

(c) MVS Medizinverlage Stuttgart GmbH \& Co. KG ISSN 0014-0082 